Check for updates

Cite this: RSC Adv., 2017, 7, 45408

Received 16th July 2017

Accepted 19th September 2017

DOI: $10.1039 / \mathrm{c} 7 \mathrm{ra07827g}$

rsc.li/rsc-advances

\section{Separation performance of dithienyl benzothiadiazole-based stationary phases for capillary gas chromatography $\dagger$}

\author{
Jianlin Peng, ${ }^{a}$ Tao Sun, ${ }^{\mathrm{ab}}$ Lianqian $\mathrm{Wu}^{\mathrm{a}}$ Meiling Qi (D) *a and Xuebin Huang (D) *a
}

\section{Introduction}

Dithienyl benzothiadiazole (TBT) derivatives have a heterocyclic $\pi$-conjugated backbone structure with electron-donor/acceptor (EDA) units and have attracted growing attention in organic solar cells and transistors. ${ }^{\mathbf{1 - 5}}$ Their unique structures and favorable physicochemical features, such as good chemical and thermal stability and solubility in organic solvents, also endow them promising potential in analytical chemistry as selective bioprobes $^{6}$ and stationary phases. ${ }^{7}$ Currently, the $\pi$-conjugated materials investigated for chromatographic separations mainly include graphene-based materials ${ }^{8-12}$ and graphitic carbon nitride. ${ }^{13}$ Differing from these materials, TBT-based materials are composed of heterocyclic dithienyl and benzothiadiazole units and have the potential as a new class of GC stationary phases. However, they are barely investigated and only one report on the TBT and TBT-C12 stationary phases is available. ${ }^{7}$ They had a column efficiency of about 1000 plates per $\mathrm{m}$ and exhibited good separation ability mainly for apolar to weakly-

${ }^{a}$ Key Laboratory of Cluster Science, Ministry of Education of China, Beijing Key Laboratory of Photoelectronic/Electrophotonic Conversion Materials, School of Chemistry and Chemical Engineering, Beijing Institute of Technology, Beijing, 100081, China.E-mail: mlqi@bit.edu.cn; huangxb@bit.edu.cn

${ }^{b}$ College of Chemistry and Chemical Engineering, Henan Key Laboratory of FunctionOriented Porous Materials, Luoyang Normal University, Luoyang, 471934, China

$\uparrow$ Electronic supplementary information (ESI) available. See DOI: $10.1039 / \mathrm{c} 7 \mathrm{ra07827g}$ polar analytes. Still, there is large room for further upgrading their selectivity and resolving capability for a wider range of analytes and their isomers. Designing new TBT-based stationary phases to achieve the above purpose is quite worth exploring for their fundamental research and practical applications in GC.

Introducing certain functional groups or altering their proportions has been the feasible strategy to develop a family of stationary phases of varying selectivity for diverse analytes. ${ }^{\mathbf{1 4 - 1 8}}$ For example, increasing the phenyl content from 5\% to $35 \%$ raises the polarity of the resulting polysiloxane and improves their separation performance for moderately polar analytes. ${ }^{\mathbf{1 9}}$ Modified cyclodextrins with allyl groups exhibited high column efficiency and separation performance for benzene isomers and enantiomers. ${ }^{15,16}$ Regarding the TBT-based materials, increasing their polarity to some extent by functionalizing the basic structure with certain substituents may further enhance their selectivity for a wide range of analytes, which is highly needed in practical GC analyses.

Herein, we present the separation performance of two new TBT derivatives, namely 4,7-di(5-allyl-2-thienyl)-benzothiadiazole (TBTAA) and 4-(5-ally-2-tienyl)-7-(5-dodecyl-2-thienyl)-benzothiadiazole (TBT-AC12) (Fig. 1) as stationary phases for capillary GC separations. Introduction of allyl groups into the structure may increase their polarity over the TBT and TBT-C12 previously reported. After their column efficiency and polarity were determined, the TBT-AA and TBT-AC12 columns were investigated for their selectivity and resolving ability by employing analytes of varying types from aliphatics (alcohols, bromoalkanes, esters, alkanes and hexane 


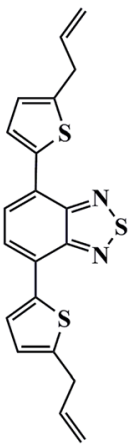

TBT-AA

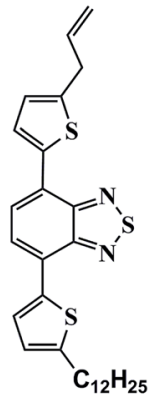

TBT-AC12
Fig. 1 Structures of TBT-AA and TBT-AC12 stationary phases.

isomers) to aromatics (benzene derivatives and their isomers including chloronitrobenzenes, dibromobenzenes, cymenes). Moreover, column repeatability and thermal stability were examined. In addition, one commercial DB-35MS column with comparable polarity was employed for comparison study.

\section{Experimental}

\subsection{Chemical and materials}

All chemicals and reagents in this work were obtained commercially with purity at least of analytical grade and used without further purification. Methyl undecanoate, trans-decahydronaphthalene, $n$-nonane, 1-octanol, benzaldehyde, $o$-dichlorobenzene, 1bromooctane, 1-nonanol, 1-bromononane, 1,5-dibromopentane, 1-decanol, $n$-pentadecane, $n$-butylbenzene, $n$-dodecane, $n$-hexane, $n$-octane, $n$-hexylbenzene, $p$-dibromobenzene, $o$-dibromobenzene, $m$-dibromobenzene, 2-methylnaphthalene, 1-methylnaphthalene, 1,3,5-trichlorobenzene, $m$-cymene, $o$-cymene, $p$-cymene, 3-methylpentane, 2,2-dimethylbutane, 2,3-dimethylbutane, $o$-chloronitrobenzene, $m$-chloronitrobenzene, $p$-chloronitrobenzene, $o$ bromonitrobenzene and $p$-bromonitrobenzene were purchased from J\&K. Scientific. Ltd. (Beijing, China). 1-Nitropropane, 1butanol, 2-pentanone, salicylaldehyde and benzyl alcohol were purchased from Aladdin Industrial Corp. (Shanghai, China). Benzene, pyridine, 1,4-dibromobutane, benzonitrile, naphthalene, dichloromethane, chloroform and methanol were purchased from the Sinopharm Chemical Reagent Co. Ltd. (Beijing, China). Dichloromethane was used as the solvent for solutes unless otherwise specified. Untreated fused-silica capillary tubing ( 0.25 $\mathrm{mm}$, i.d.) was purchased from Yongnian Ruifeng Chromatogram Apparatus Co., Ltd. (Hebei, China). A DB-35MS capillary column (10 m long $\times 0.25 \mathrm{~mm}$ i.d., film thickness $0.25 \mu \mathrm{m}$ ) coated with 35\% phenyl methylpolysiloxane was purchased from Agilent Technologies (California, USA) for comparison study.

\subsection{Instruments}

GC chromatograms were recorded on an Agilent 7890A gas chromatograph (Agilent Technologies, California, USA), equipped with a split/splitless injector, an autosampler and a flame ionization detector (FID). Data analysis was carried out by ChemStation software. Nitrogen with high purity (99.999\%) was used as the carrier gas. Unless otherwise specified, GC conditions for separations were performed as follows: injection volume of $0.1 \mu \mathrm{L}$, injection port at $250{ }^{\circ} \mathrm{C}$, split ratio of $50: 1$, flow rate of $1 \mathrm{~mL} \mathrm{~min}^{-1}$, and FID at $300{ }^{\circ} \mathrm{C}$. The oven temperature programs for the separations are individually provided in the following figure captions.

NMR spectra were recorded on a Bruker Avance-400NMR spectrometer (Bruker, Switzerland). ESI-MS data was obtained on Bruker BIFLEX III (Bruker, Switzerland). Thermal gravimetric analysis (TGA) was performed on a DTG-60AH thermal gravimetric analyzer (Shimadzu, Japan) from $30{ }^{\circ} \mathrm{C}$ to $600{ }^{\circ} \mathrm{C}$ at a ramp of $10{ }^{\circ} \mathrm{C} \mathrm{min}^{-1}$ under nitrogen. Scanning electron microscopy (SEM) images were recorded on a Hitachi S4800 microscope (Tokyo, Japan).

\subsection{Synthesis of the TBT-AA and TBT-AC12 stationary phases $^{3,20,21}$}

5-Dodecylthiophen (0.46 g, $1.82 \mathrm{mmol})$ in anhydrous THF (5 $\mathrm{mL}$ ), $n$-BuLi (2.5 $\mathrm{M}$ in hexane, $0.75 \mathrm{~mL}, 1.88 \mathrm{mmol}$ ) was added dropwise under a nitrogen atmosphere. The mixture was stirred at $-78{ }^{\circ} \mathrm{C}$ and $\mathrm{ZnCl}_{2}(0.36 \mathrm{~g}, 2.7 \mathrm{mmol}$, dissolved in $3 \mathrm{~mL} \mathrm{THF})$ was added dropwise. After stirring for $1.5 \mathrm{~h}$ at the same temperature, the mixture was warmed to room temperature to evaporate the solvent. Thus, (5-dodecyl-2-thienyl)-zinc chloride (compound 1) was obtained as yellow oil and used for the following synthesis. Similarly, (5-allyl-2-thienyl)-zinc chloride (compound 2) was synthesized from 5-allylthiophene $(0.23 \mathrm{~g}$, $1.85 \mathrm{mmol})$ and $n$-BuLi (2.5 $\mathrm{M}$ in hexane, $0.75 \mathrm{~mL}, 1.88 \mathrm{mmol})$.

The compound 1, compound 2, 4,7-dibromo-2,1,3benzothiadiazole $(0.14 \mathrm{~g}, 0.5 \mathrm{mmol})$ and $\mathrm{Pd}\left(\mathrm{PPh}_{3}\right)_{4}(0.02 \mathrm{~g}, 0.03$ $\mathrm{mmol}$ ) were dissolved in $25 \mathrm{~mL}$ of dry and degassed THF. After stirring for $12 \mathrm{~h}$ at $65{ }^{\circ} \mathrm{C}$, the solvent was evaporated and the product was purified by column chromatography (silica gel, petroleum ether/dichloromethane (20:1) as eluent). As a result, the products of TBT-AA (32.2 $\mathrm{mg}, 0.084 \mathrm{mmol}$, yield $16.9 \%)$ and TBT-AC12 (75.4 mg, $0.148 \mathrm{mmol}$, yield 29.6\%) were obtained as orange solids and characterized by ${ }^{1} \mathrm{H}-\mathrm{NMR},{ }^{13} \mathrm{C}-\mathrm{NMR}$ and ESIMS, respectively. The obtained data for TBT-AA are: ${ }^{1} \mathrm{H}-\mathrm{NMR}$ (400 MHz, $\left.\mathrm{CDCl}_{3}, \delta \mathrm{ppm}\right)$ : $7.95-7.94(\mathrm{~d}, 2 \mathrm{H}), 7.77$ (s, 2H), 6.916.90 (d, 2H), 6.15-5.90 (m, 2H), 5.26-5.16 (m, 4H), 3.66-3.64 (d, $4 \mathrm{H}) ;{ }^{13} \mathrm{C}-\mathrm{NMR}\left(400 \mathrm{MHz}, \mathrm{CDCl}_{3}, \delta \mathrm{ppm}\right): 152.7,144.8,137.8$, 136.2, 127.6, 126.0, 125.3, 116.8, 109.9, 34.7; ESI-MS: $\mathrm{m} / \mathrm{z}$ calcd for $\mathrm{C}_{20} \mathrm{H}_{16} \mathrm{~N}_{2} \mathrm{~S}_{3}$ : 380.0476 , found: $380.0482\left(\mathrm{M}^{+}\right)$. The obtained data for TBT-AC12 are: ${ }^{1} \mathrm{H}-\mathrm{NMR}\left(400 \mathrm{MHz}, \mathrm{CDCl}_{3}, \delta \mathrm{ppm}\right) 7.93-7.92(\mathrm{t}$, $2 \mathrm{H}), 7.74$ (s, 2H), 6.90-6.86 (dd, 2H), 6.11-6.01 (m, 1H), 5.26-5.22 (dd, 1H), 5.19-5.16 (dd, 1H), 3.65-3.64 (d, 2H), 2.90-2.86 (t, 2H), 1.42-1.37 (m, 20H), 0.91-0.87 (t, 3H); ${ }^{13} \mathrm{C}-\mathrm{NMR}$ (400 MHz, $\mathrm{CDCl}_{3}$, $\delta$ ppm) 152.7, 147.9, 144.7, 137.9, 136.9, 136.2, 127.5, 126.0, 125.9, 125.7, 125.3, 125.1, 116.8, 34.7-14.3; ESI-MS: $\mathrm{m} / \mathrm{z}$ calcd for $\mathrm{C}_{29} \mathrm{H}_{36} \mathrm{~N}_{2} \mathrm{~S}_{3}$ : 508.2041, found: $508.2048\left(\mathrm{M}^{+}\right)$.

\subsection{Preparation of the TBT-AA and TBT-AC12 capillary columns}

The as-synthesized TBT-AA and TBT-AC12 stationary phases were individually dissolved in dichloromethane $(0.15 \%, \mathrm{w} / \mathrm{v})$ and coated onto a fused-silica capillary column $(10 \mathrm{~m}$ long, 
$0.25 \mathrm{~mm}$, i.d.) by static coating method, respectively. Prior to coating, the capillary column was pretreated with a saturated solution of sodium chloride in methanol and kept for $2 \mathrm{~h}$ to deposit the microcrystals of sodium chloride on the inner wall with an aim to roughen the inner surface and facilitate subsequent coating with a stationary phase..$^{22}$ Then, the solution was removed and the column was conditioned from $40{ }^{\circ} \mathrm{C}$ to $200{ }^{\circ} \mathrm{C}$ at $10{ }^{\circ} \mathrm{C} \mathrm{min}{ }^{-1}$ and held for $3 \mathrm{~h}$ under nitrogen. Afterwards, the pretreated column was ready for the subsequent coating. The coating solution of the given stationary phase was introduced into the capillary column at $40{ }^{\circ} \mathrm{C}$ and then the column was sealed on one end and connected to vacuum system on the other end to slowly evaporate the solvent. After that, the column was conditioned from $40{ }^{\circ} \mathrm{C}$ held for $30 \mathrm{~min}$ to $180{ }^{\circ} \mathrm{C}$ at $1{ }^{\circ} \mathrm{C} \mathrm{min}$ mi $^{-1}$ and held at $180{ }^{\circ} \mathrm{C}$ for $7 \mathrm{~h}$ under nitrogen. The coating thickness of the obtained column was calculated as about $0.1 \mu \mathrm{m}$ by the empirical formula, $d_{\mathrm{f}}=\left(d_{\mathrm{c}} \times c\right) / 400$, where $d_{\mathrm{c}}$ is the capillary inner diameter $(\mu \mathrm{m}), c$ is the concentration of the stationary phase $(\%, w / v)$.

\section{Results and discussion}

\subsection{Characterization of TBT-AA and TBT-AC12 stationary phases and columns}

TGA was employed to evaluate the intrinsic thermal stability of TBT-AA and TBT-AC12 stationary phases. As shown in Fig. 2A, the temperature where $5 \%$ weight loss existed was $290{ }^{\circ} \mathrm{C}$ for TBT-AA and $325{ }^{\circ} \mathrm{C}$ for TBT-AC12, respectively, suggesting their good thermal stability as GC stationary phases. Fig. 2B presents the Golay plots of the TBT-AA and TBT-AC12 columns determined by $n$-dodecane under $100{ }^{\circ} \mathrm{C}$, showing the minimum HETP of $0.269 \mathrm{~mm}$ at $0.40 \mathrm{~mL} \mathrm{~min}^{-1}(k=4.30)$ and $0.263 \mathrm{~mm}$ at $0.30 \mathrm{~mL} \min ^{-1}(k=6.75)$, respectively. Correspondingly, the maximum column efficiency was 3700 plates per $m$ for the TBTAA column and 3800 plates per $m$ for the TBT-AC12 column. Moreover, Fig. 2C and D provide the SEM images on the inner wall surface of the TBT-AA and TBT-AC12 columns, respectively, showing relatively regular patterns of the stationary phases interlaced with some gaps.

Polarity of a stationary phase is closely related with its selectivity for solutes via different intermolecular interactions and is often evaluated by McReynolds constants. The McReynolds constants of the TBT-AA and TBT-AC12 stationary phases were determined under $120{ }^{\circ} \mathrm{C}$ at flow rate of $1.0 \mathrm{~mL} \mathrm{~min}^{-1}$ by using the five probing solutes of benzene $\left(X^{\prime}\right)$, 1-butanol $\left(Y^{\prime}\right), 2$ pentanone $\left(Z^{\prime}\right)$, 1-nitropropane $\left(U^{\prime}\right)$ and pyridine $\left(S^{\prime}\right)$. Table 1 summarizes the obtained McReynolds constants of the stationary phases. The average values of 186 for TBT-AA and 123 for TBT-AC12 suggested their moderate polarity close to that of the DB-35MS phase. Clearly, the TBT-AA and TBT-AC12 stationary phases have higher polarity with the introduction of the allyl groups than the TBT (average $=98$ ) and TBT-C12 (average $=36$ ) stationary phases without the group, ${ }^{7}$ respectively. In addition, the TBT-AA stationary phase displays much higher $Y^{\prime}, U^{\prime}$ and $S^{\prime}$ values than the TBT-AC12 phase, indicating that they may differ in retention behaviours and resolving performance for GC separations.
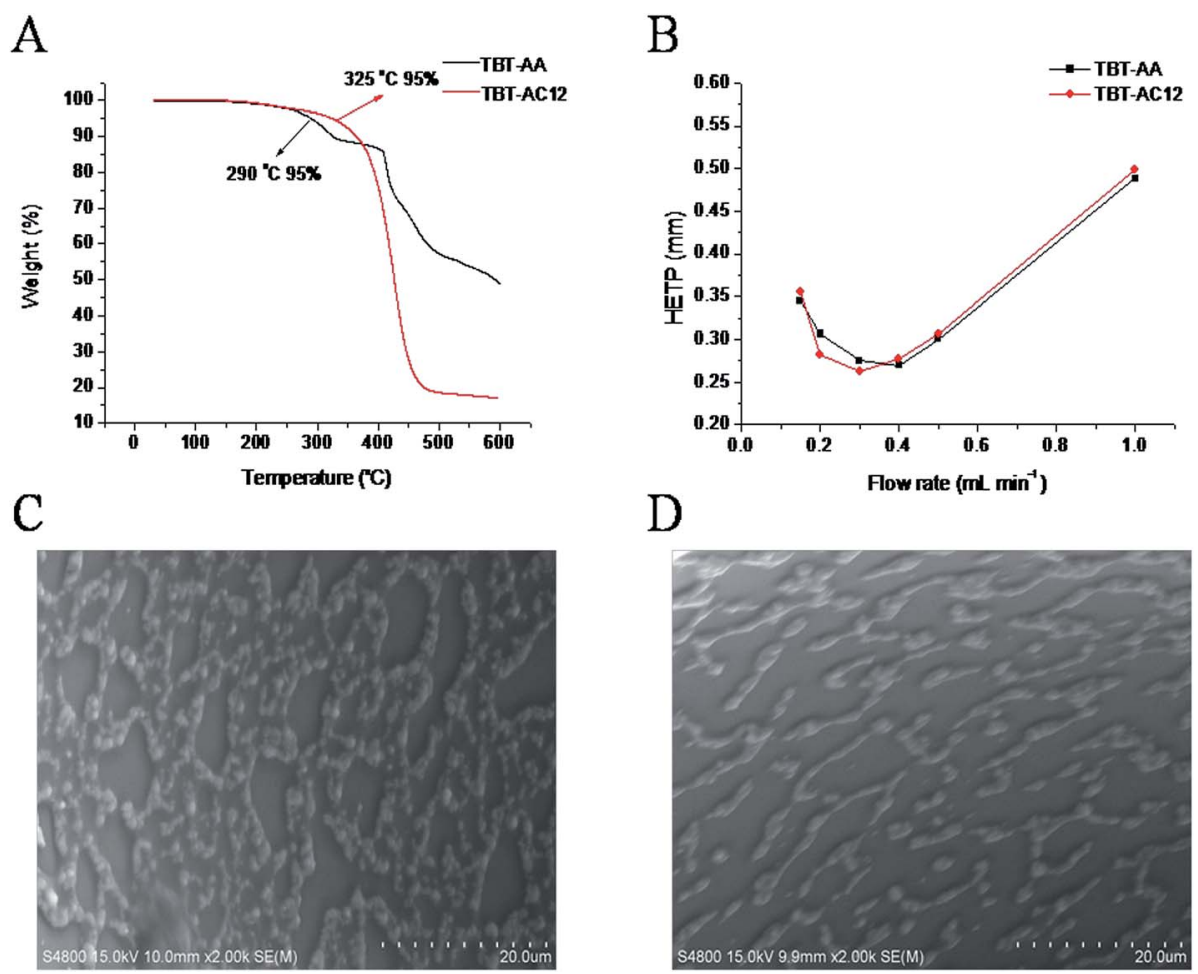

Fig. 2 (A) TGA curves of the TBT-AA and TBT-AC12 stationary phases; (B) Golay plots determined by $n$-dodecane under $100{ }^{\circ} \mathrm{C}$; (C) and (D) SEM images showing the inner coating of the TBT-AA and TBT-AC12 columns, respectively. 
Table 1 McReynolds constants of the TBT-AA, TBT-AC12 and DB-35MS stationary phases ${ }^{a}$

\begin{tabular}{|c|c|c|c|c|c|c|c|}
\hline Stationary phase & $X^{\prime}$ & $Y^{\prime}$ & $Z^{\prime}$ & $U^{\prime}$ & $S^{\prime}$ & General polarity & Average polarity \\
\hline$I$ for TBT-AA & 773 & 765 & 781 & 890 & 942 & & \\
\hline$I$ for TBT-AC12 & 733 & 707 & 728 & 809 & 857 & & \\
\hline$\Delta I$ for TBT-AA & 120 & 175 & 154 & 238 & 243 & 930 & 186 \\
\hline$\Delta I$ for TBT-AC12 & 80 & 117 & 101 & 157 & 158 & 613 & 123 \\
\hline$\Delta I$ for DB-35MS & 102 & 142 & 145 & 219 & 178 & 786 & 157 \\
\hline
\end{tabular}

\subsection{Separation performance of the TBT-AA and TBT-AC12 stationary phases}

The aim of this work was to design new TBT-based stationary phases with high resolving capability for analytes of wide ranging polarity to address the problem with the previous TBT and TBT-C12 phases that showed limited selectivity to analytes of low polarity. In the present work, to comprehensively evaluate the resolving capability of the two new TBT-based stationary phases, we employed the mixtures consisting of analytes ranging wide polarity and types, and also the mixtures of positional and structural isomers of high resemblance in their physicochemical properties.

First, a mixture of 11 analytes of diverse types was separated on the TBT-AA and TBT-AC12 columns in comparison to the DB35MS column. As can be seen from Fig. 3, the TBT-based columns baseline resolved all of the analytes, displaying good separation performance for analytes ranging from apolar to polar nature. In contrast, three pairs of the analytes were overlapped mostly or even coeluted on the commercial column, i.e., trans-decahydronaphthalene/benzaldehyde (peaks 1/2), 1,5dibromopentane/1-decanol (peaks 8/9) and methyl undecanoate/pentadecane (peaks 10/11). Their high-resolution performance over the conventional stationary phase may originate from their unique retention behaviors for diverse analytes. Also, these two TBT-based stationary phases differ in their specific retention behaviors. Compared with the TBT-AA, the TBT-AC12 stationary phase retained longer for the analytes with a long alkyl chain, including apolar alkanes, weakly polar 1bromoalkanes (1-bromononane, 1-bromooctane, methyl undecanoate) and polar alcohols (1-octanol, 1-nonanol, 1-decanol). This interesting phenomenon may be ascribed to the better shape-matching effect between the long alkyl group of these analytes and the $\mathrm{C} 12$ chain of the stationary phase, leading to their stronger van der Waals interactions with the phase. Particularly, the unusually prolonged retention of the polar alcohols on the less polar TBT-AC12 column confirmed the enhanced cooperative effect of van der Waals forces and $\mathrm{H}$ bonding interactions by the shape-fitting effect. Generally, the TBT-based columns exhibited longer retention for the alcohols than the DB-35MS column that showed the reversal elution of 1,5-dibromopentane/1-decanol (peak 8/9) with incomplete resolution $(R=0.84)$. Additionally, the prolonged retention of 1bromooctane and 1-bromononane on the TBT-AC12 column resulted in the reversal elution of the peaks $4 / 5$ and a narrower space between the peaks $7 / 8$ in comparison to the TBT-AA column. Besides, the TBT-AC12 column reversed the elution of the peaks $1 / 2$, stemming from its stronger dispersion interaction with the nonpolar trans-decahydronaphthalene.

In the light of the heterocyclic $\pi$-conjugated structure of the TBT-based stationary phases, their separation capability for aromatic analytes was quite worth exploring. Accordingly, another mixture mainly containing benzene and naphthalene derivatives was utilized for the investigation. As shown in Fig. 4, the TBT-AC12 column achieved baseline separation of all the analytes with good peak shapes and showed advantageous
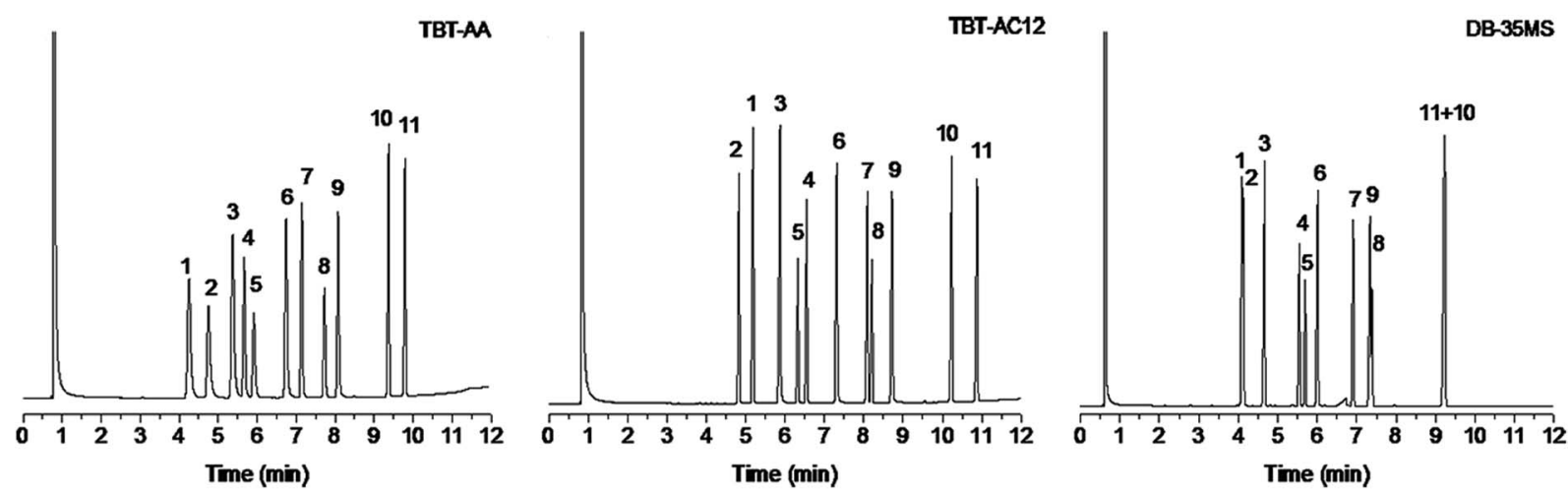

Fig. 3 Separation of the mixture containing 11 analytes of diverse types on the indicated columns under the temperature program of $40{ }^{\circ} \mathrm{C}$ to $150^{\circ} \mathrm{C}$ at $10^{\circ} \mathrm{C} \mathrm{min}^{-1}$. Peaks: (1) trans-decahydronaphthalene, (2) benzaldehyde, (3) 1-octanol, (4) 1-bromooctane, (5) 1,4-dibromobutane, (6) 1 nonanol, (7) 1-bromononane, (8) 1,5-dibromopentane, (9) 1-decanol, (10) methyl undecanoate, (11) $n$-pentadecane. 

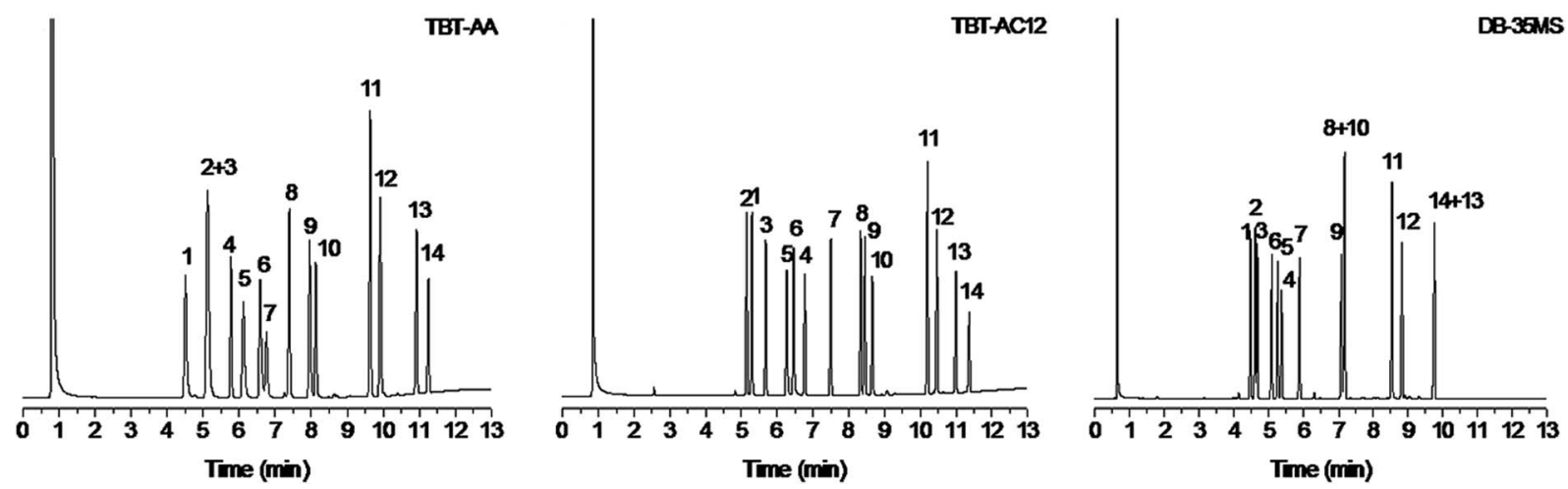

Fig. 4 Separations of the mixture of 14 analytes of diverse types on the indicated three columns under the temperature program of $40{ }^{\circ} \mathrm{C}$ to $150{ }^{\circ} \mathrm{C}$ at $10^{\circ} \mathrm{C} \mathrm{min}^{-1}$. Peaks: (1) $n$-butylbenzene, (2) benzonitrile, (3) o-dichlorobenzene, (4) $n$-dodecane, (5) salicylaldehyde, (6) benzyl alcohol, (7) 1,3,5-trichlorobenzene, (8) $n$-hexylbenzene, (9) naphthalene, (10) $p$-dibromobenzene, (11) 2-methylnaphthalene, (12) 1-methylnaphthalene, (13) o-bromonitrobenzene, (14) p-bromonitrobenzene.

performance for the aromatics over the other two columns. Observably, the TBT-AA column coeluted benzonitrile and $o$ dichlorobenzene (peaks 2/3) whereas the DB-35MS column overlapped the analytes of $n$-hexylbenzene/ $p$-dibromobenzene (peaks 8/10) and $o$-bromonitrobenzene/ $p$-bromonitrobenzene (peaks 13/14). In contrast, the TBT-AC12 column well resolved the peaks $2 / 3$ by its stronger $\pi-\pi$ and halogen-bonding interactions with $o$-dichlorobenzene. Moreover, the complete separation of the peaks $8 / 10$ and $13 / 14$ on the TBT-based columns can be credited to their stronger interactions with the halogenated benzenes of $p$-dibromobenzene, $o$-bromonitrobenzene and $p$-bromonitrobenzene through $\pi-\pi$, halogen-bonding and dipole-dipole interactions.

For the aromatics, the TBT-based columns exhibited different retention behaviours from the DB-35MS column. Noticeably, they exhibited prolonged retention for benzyl alcohol, alkylated or halogenated benzenes and naphthalenes owing to their comprehensive interactions with the analytes via H-bonding, $\pi-\pi, \pi-\pi$ EDA, halogen-bonding and van der Waals forces. Credited by these interactions, the TBT-based stationary phases are capable of well resolving the peaks 8/10 and 13/14 and reversing the elution of salicylaldehyde/benzyl alcohol (peaks 5/6) and $n$-hexylbenzene/naphthalene (peaks 8/9) in contrast to the polysiloxane phase. Noteworthy, they eluted the analytes of $n$-hexylbenzene/naphthalene (peaks $8 / 9$, bp $226^{\circ} \mathrm{C} /$ $218{ }^{\circ} \mathrm{C}$ ), $o$-bromonitrobenzene/ $p$-bromonitrobenzene (peaks $13 / 14$, bp $261^{\circ} \mathrm{C} / 256^{\circ} \mathrm{C}$ ) in a reversal order against their boiling points. These phenomena may individually originate from the stronger $\pi-\pi$ interaction of naphthalene and from the slightly weaker interactions of $o$-bromonitrobenzene than its $p$-isomer because of its intramolecular halogen-bonding interaction..$^{23}$ In addition, the two TBT columns differed in their elution order for the analytes of $n$-butylbenzene/benzonitrile (peaks $1 / 2$ ) and dodecane/salicylaldehyde (peaks 4/5), evidencing their different retention behaviors. Comparatively, the TBT-AC12 stationary phase prolonged the retention of $n$-butylbenzene and $n$-dodecane, especially for $n$-dodecane, which further proves its shapefitting selectivity as stated above. Moreover, comparison with the TBT and TBT-C12 stationary phases in our previous work ${ }^{7}$ reveals that the present TBT-AA and TBT-AC12 stationary phases exhibit higher separation capability for polar analytes such as alcohols and halogenated nitrobenzenes possibly due to their increased polarity by the allyl groups. This finding suggests that the separation performance of TBT-based materials could be tuned by varying their chemical structures and functional groups.

The above findings on their preferential retention and selectivity for halogenated and alkylated benzenes encouraged us to further explore their potential for isomer mixtures that are hard to be well resolved by conventional GC stationary phases. Fig. 5 shows the separation results for the isomer mixtures of chloronitrobenzenes, dibromobenzenes and cymenes on the three columns. As can be seen, the TBT columns generally achieved higher resolution of the isomers than the DB-35MS column. A close inspection of the results can find that the TBT-AA stationary phase exhibits increasing resolving capability for the analytes with stronger electron-withdrawing groups while the TBT-AC12 phase behaves in the opposite trend. Clearly, the TBT-AA and TBT-AC12 columns completely resolved the isomers of chloronitrobenzenes and cymenes in a sharp contrast to their partial resolution $(R=0.83$ for $p$-/o-chloronitrobenzene, $R=0.92$ for $m$ - $/ p$-cymene) on the DB-35MS column.

It is important to elucidate the probable retention mechanism behind the high-resolution performance of the TBT-based stationary phases. For the chloronitrobenzene isomers in Fig. 5A, D and G, the TBT-AA column baseline resolved all of them whereas the TBT-AC12 and DB-35MS columns partially separated the $m$ - $/ o$-isomers $(R=1.17)$ and $p$-/o-isomers $(R=$ $0.83)$, respectively. The results demonstrate the dramatic effect of allyl groups on the improvement of resolving performance. Moreover, it is interesting to note that the TBT columns displayed the reversal elution of the $o$ - $/ p$-isomers $\left(\mathrm{bp} 246^{\circ} \mathrm{C} / 242^{\circ} \mathrm{C}\right.$ ) against the order of their boiling points, differing from that on the DB-35MS column. The longer retention of $p$-chloronitrobenzene than its $o$-isomer evidenced the major contribution of $\pi-\pi$ EDA and halogen-bonding interactions to the retention 

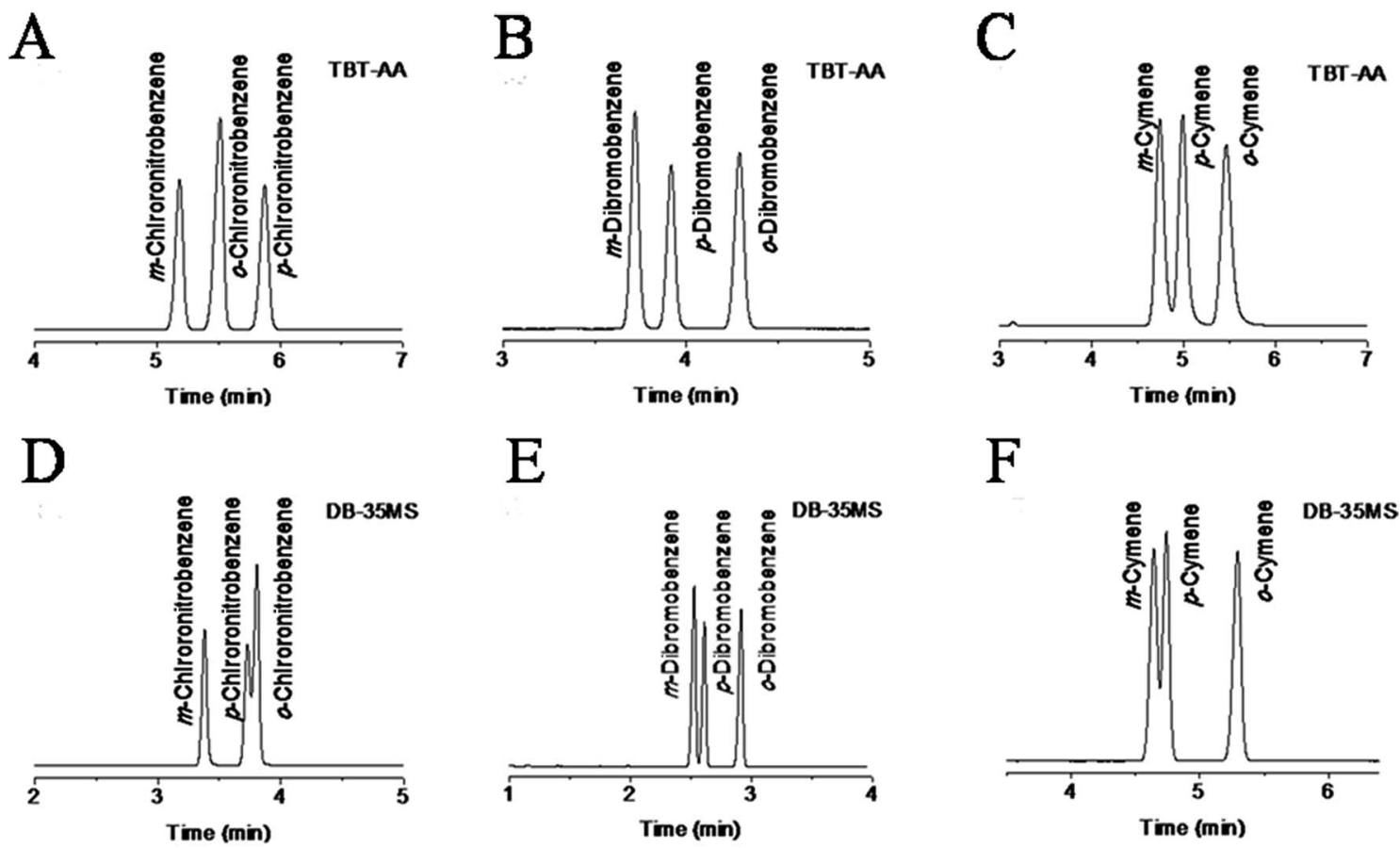

$\mathrm{F}$
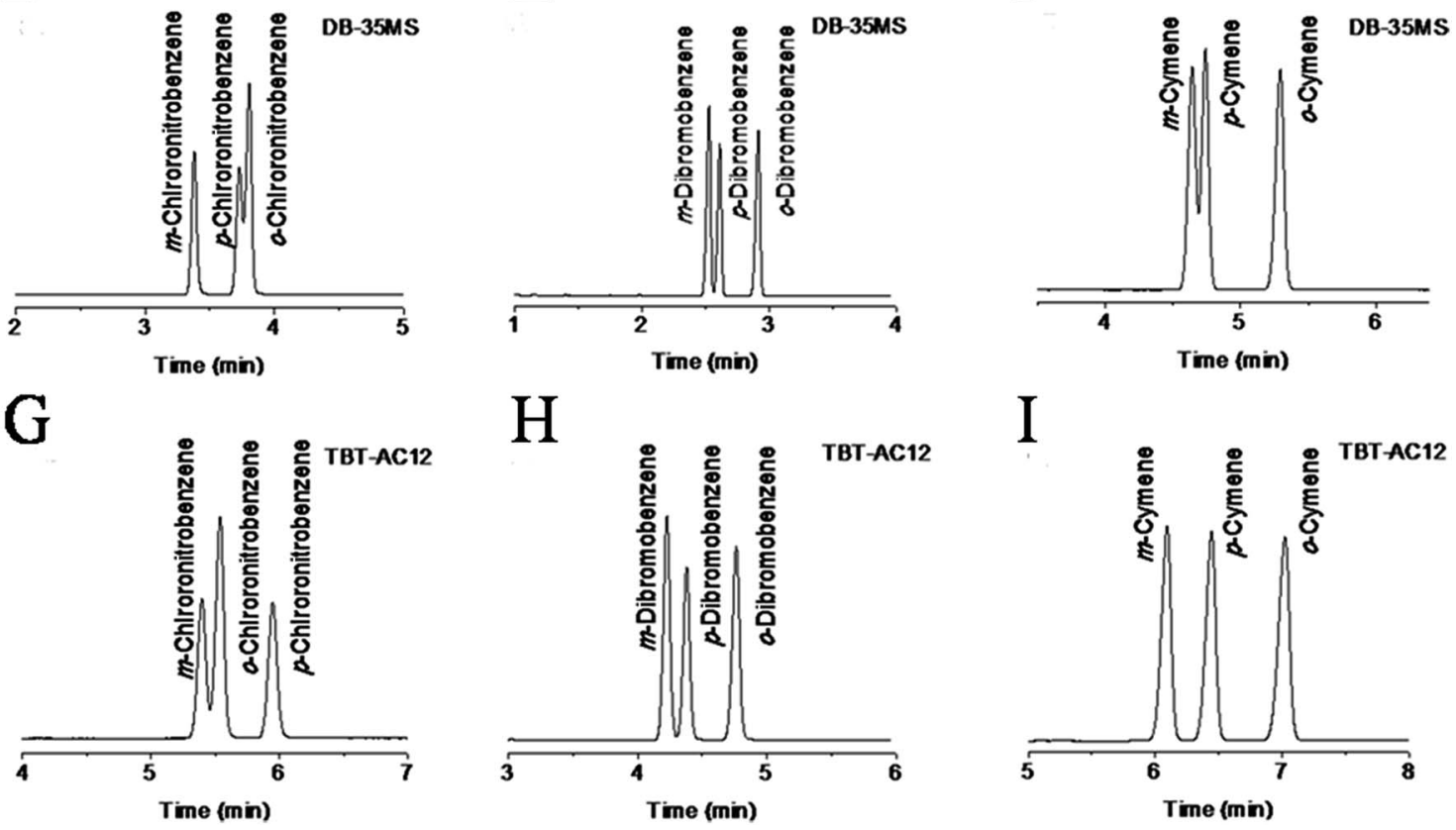

Fig. 5 Separations of the isomer mixtures of chloronitrobenzenes $(A, D, G)$, dibromobenzenes $(B, E, H)$ and cymenes $(C, F, I)$ on the indicated columns. GC conditions: $130{ }^{\circ} \mathrm{C}$ for chloronitrobenzenes and dibromobenzenes and $75{ }^{\circ} \mathrm{C}$ for cymenes at the flow rate of $0.5 \mathrm{~mL}$ min ${ }^{-1}$.

and separation of the TBT stationary phases. Relatively, the benzene ring of $p$-chloronitrobenzene is more electron-deficient than its $o$-isomer, leading to its stronger $\pi-\pi$ EDA interaction with the electron-rich TBT stationary phases. In addition, the strong electron-withdrawing nitro group at the para-position makes the $\delta^{+}$hole on the chlorine atom more positive,$^{24}$ resulting in its stronger halogen-bonding interaction with the TBT stationary phases.

For the dibromobenzene isomers in Fig. 5B, E and $\mathrm{H}$, the TBT columns also achieved complete separations. In particular, for the critical pair of $m$ - $/ p$-isomers (bp $218^{\circ} \mathrm{C} / 219^{\circ} \mathrm{C}$ ), the TBTAA and TBT-AC12 columns still attained higher resolution of 2.36 and 1.61 , respectively, than that $(R=1.46)$ on the DB-35MS column. Also, the TBT-based columns well resolved the cymene isomers, as shown in Fig. 5C, F and I. For the critical pair of $m-/$ $p$-isomers (bp $176{ }^{\circ} \mathrm{C} / 178{ }^{\circ} \mathrm{C}$ ), the corresponding resolution values for the TBT-AC12, TBT-AA and DB-35MS columns were $2.43,1.45$ and 0.92 , respectively. The above results clearly evidenced the advantageous separation performance of the TBT stationary phases, mainly deriving from their unique structures and specific molecular interactions commented above. Inspired by the high-resolution performance of the TBT-AC12 column for the apolar isomers and its preferential retention for alkanes, we investigated its separation ability for the hexane isomers in comparison to the DB-35MS column (Fig. S1 in the ESI $\dagger$ ). As a result, the TBT-AC12 column achieved the resolution of 2.35, 1.67 and 1.96 for the isomers by their elution order, higher than the corresponding values $(1.94,1.36$ and 1.52) on the DB-35MS column. Undoubtedly, the TBT-AC12 stationary phase exhibits high distinguishing capability for both aliphatic and aromatic hydrocarbon isomers. The elution of the hexane isomers basically follows the order of their van der Waals surface areas (144.6, 145.9, 156.9 and $\left.160.4 \mathrm{pm}^{2} \times 10^{-4}\right),{ }^{25}$ i.e., the larger surface area, the longer retention on the columns.

\subsection{Column repeatability and thermal stability}

For the TBT-based columns, their column repeatability and reproducibility concerning run-to-run, day-to-day and columnto-column were determined by separations of the isomer mixtures of chloronitrobenzenes and dibromobenzenes on the 
TBT-AA columns and cymenes and hexanes on the TBT-AC12 columns, and were evaluated by the relative standard deviation (RSD) values on their retention times. Using the isomers for the evaluation is more demanding since isomer separations are more sensitive to the slight variations of the column performance. The obtained results are summarized in Tables S1 and S2 (ESI $\dagger$ ) for the TBT-AA and TBT-AC12 columns, respectively. The obtained RSD values are $0.02-0.12 \%$ for run-to-run, $1.3-$ $1.8 \%$ for day-to-day and $4.7-5.0 \%$ for column-to-column for the TBT-AA column and the corresponding values for the TBT-AC12 column are $0.03-0.09 \%, 1.1-1.3 \%$ and $1.7-1.9 \%$. The above results demonstrate their good column repeatability and reproducibility for GC separations.

Their column thermal stability was measured by separations of the chloronitrobenzene isomers and cymene isomers on the TBT-AA and TBT-AC12 columns, respectively, after the columns were conditioned up to different temperatures (TBT-AA: $180{ }^{\circ} \mathrm{C}$, $190{ }^{\circ} \mathrm{C}, 200^{\circ} \mathrm{C}, 210^{\circ} \mathrm{C}$ each for $0.5 \mathrm{~h}$; TBT-AC12 : $180{ }^{\circ} \mathrm{C}, 200^{\circ} \mathrm{C}$, $210{ }^{\circ} \mathrm{C}, 220{ }^{\circ} \mathrm{C}, 230{ }^{\circ} \mathrm{C}$ each for $1 \mathrm{~h}$, respectively). The relative standard deviation (RSD) values on retention times were 4.5$4.8 \%$ for chloronitrobenzene isomers on TBT-AA column after conditioned up to $200{ }^{\circ} \mathrm{C}$ and the corresponding resolution for $p$-/o-chloronitrobenzene was 1.55. After conditioned up to $220^{\circ} \mathrm{C}$, the relative standard deviation (RSD) values of retention times were $10 \%$ for cymene isomers on TBT-AC12 column and the resolution of $p$-/m-cymene obtained was 1.65. As a result, the maximum operating temperature was recommended as $200{ }^{\circ} \mathrm{C}$ for the TBT-AA column and $220{ }^{\circ} \mathrm{C}$ for the TBT-AC12 column, respectively. Introduction of an allyl group into the structure greatly improves the separation performance but also affects the column thermal stability to some extent due to their instability at high temperatures.

\subsection{Application for purity determination of real samples}

To examine the potential of the TBT-AA and TBT-AC12 columns for practical use, we applied them to determine the possible isomer impurities in the reagent $o$-dibromobenzene of analytical-grade while the DB-35MS column was also used for comparison. As shown in Fig. 6, both TBT-based columns can well resolve the isomer impurity of $p$-dibromobenzene from the
Table 2 Purity determination of the reagent 0 -dibromobenzene on the indicated columns

\begin{tabular}{llll}
\hline Column & $\begin{array}{l}\text { Labeled } \\
\text { purity (\%) }\end{array}$ & $\begin{array}{l}\text { Measured } \\
\text { purity (\%) }\end{array}$ & $\begin{array}{l}\text { Isomer } \\
\text { impurity }^{a}(\%)\end{array}$ \\
\hline TBT-AA & $>98$ & 99.54 & 0.034 \\
TBT- & $>98$ & 99.52 & 0.035 \\
AC12 & $>98$ & 99.53 & 0.046 \\
DB-35MS & $>$ & \\
${ }^{a} p$-Dibromobenzene. & &
\end{tabular}

main component of $o$-dibromobenzene. In addition, the assay results of the isomers calculated by peak area normalization method are listed in Table 2. As can be seen, the results on the TBT-based columns are quite close to those on the commercial column. The above results suggest the good potential of the TBT-based columns for GC analysis of real samples.

\section{Conclusions}

This work presents the investigation of two novel TBT-based stationary phases, namely TBT-AA and TBT-AC12, for GC separations. As demonstrated, these two stationary phases exhibit greatly improved column efficiency and resolving capability for analytes of a wide ranging nature in comparison to the previous TBT stationary phases and also show advantageous performance for positional and structural isomers over the common polysiloxane phase. Their high distinguishing capability and different retention behaviours from the polysiloxane phase mainly stem from their comprehensive molecular interactions, involving $\pi-\pi$ and $\pi-\pi$ EDA, halogen-bonding, H-bonding interactions and van der Waals forces. Moreover, the new TBT stationary phases differ from each other to some extent in their selectivity and retention preferences. The TBT-AC12 stationary phase exhibits preferential retention for the analytes with a long alkyl chain and higher selectivity for alkylated benzene isomers while the TBT-AA phase shows higher selectivity for halogenated benzene isomers. This work provides an alternative class of TBT-based GC stationary phases and also presents a good example for designing a stationary phase of high selectivity.
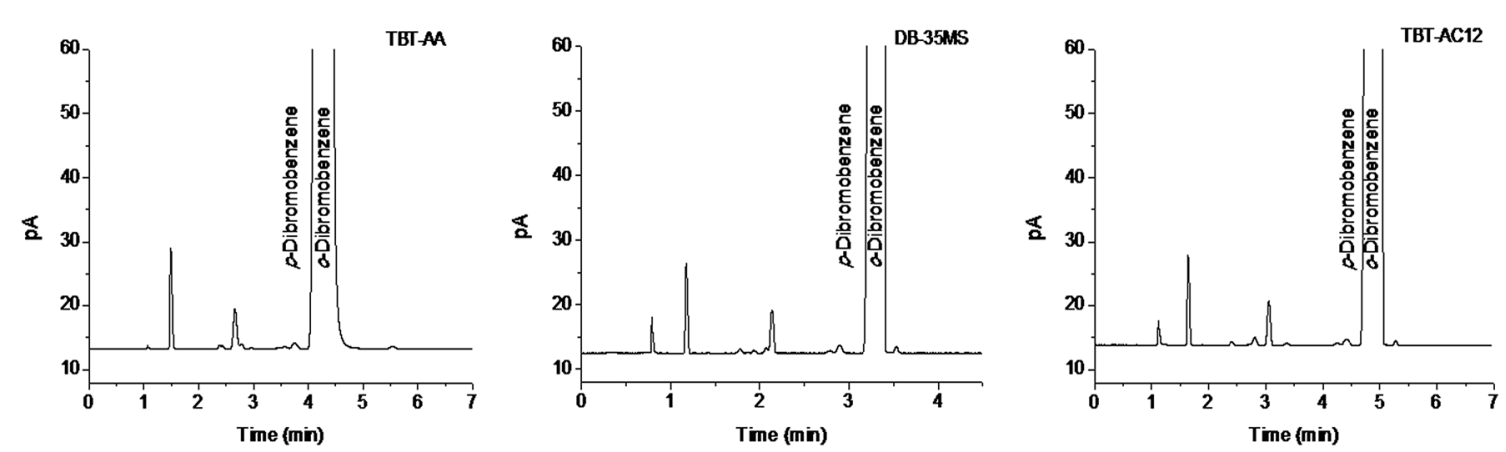

Fig. 6 Determination of the isomer impurities of the reagent 0 -dibromobenzene on the indicated columns. GC conditions: injection volume of $0.1 \mu \mathrm{L}$ of the sample without dilution, split ratio of $150: 1$, flow rate of $0.5 \mathrm{~mL} \mathrm{~min}^{-1}$, oven temperatures of $130{ }^{\circ} \mathrm{C}$ for the TBT-AA and TBT-AC12 columns, and $125^{\circ} \mathrm{C}$ for the DB-35MS column, respectively. 


\section{Conflicts of interest}

There are no conflicts to declare.

\section{Acknowledgements}

The authors are grateful for the financial support by the National Natural Science Foundation of China (21575013) and the 111 Project B07012 in China.

\section{References}

1 H. X. Zhou, L. Q. Yang, S. Q. Xiao, S. B. Liu and W. You, Macromolecules, 2010, 43, 811-820.

2 B. Liu, A. Najari, C. Y. Pan, M. Leclerc, D. Q. Xiao and Y. P. Zou, Macromol. Rapid Commun., 2010, 31, 391-398.

3 B. Y. Fu, J. Baltazar, Z. K. Hu, A. T. Chien, S. Kumar, C. L. Henderson, D. M. Collard and E. Reichmanis, Chem. Mater., 2012, 24, 4123-4133.

4 J. Z. Ren, X. C. Bao, L. L. Han, J. X. Wang, M. Qiu, Q. Q. Zhu, T. Hu, R. Y. Sheng, M. L. Sun and R. Q. Yang, Polym. Chem., 2015, 6, 4415-4423.

5 H. Mori, H. Nonobe and Y. Nishihara, Polym. Chem., 2016, 7, 1549-1558.

6 B. A. Neto, P. H. Carvalho and J. R. Correa, Acc. Chem. Res., 2015, 48, 1560-1569.

7 T. Sun, L. Tian, J. M. Li, M. L. Qi, R. N. Fu and X. B. Huang, J. Chromatogr. A, 2013, 1321, 109-118.

8 A. Speltini, D. Merli and A. Profumo, Anal. Chim. Acta, 2013, 783, 1-16.

9 J. Fan, M. L. Qi, R. N. Fu and L. T. Qu, J. Chromatogr. A, 2015, 1399, 74-79.
10 M. L. Zhang and H. D. Qiu, Trends Anal. Chem., 2015, 65, 107-121.

11 Y. B. Li, R. Z. Zhang, T. Wang, Y. H. Wang, Y. H. Wang, L. F. Li, W. J. Zhao, X. Z. Wang and J. K. Luo, Talanta, 2016, 154, 99-108.

12 X. H. Yang, C. X. Li, M. L. Qi and L. T. Qu, $R S C A d v ., 2017,7$, 32126-32132.

13 Y. Z. Zheng, Q. Han, M. L. Qi and L. T. Qu, J. Chromatogr. A, 2017, 1496, 133-140.

14 Z. R. Zeng, N. Guan, X. H. Tang and X. R. Lu, Analyst, 2000, 125, 843-848.

15 M. M. Yin, N. Li, X. Y. Shi and M. Wang, Chromatographia, 2003, 58, 301-305.

16 X. Y. Shi, P. Liang and X. W. Gao, Anal. Chim. Acta, 2005, 548, 86-94.

17 V. Schurig, Ann. Pharm. Fr., 2010, 68, 82-98.

$18 \mathrm{X}$. Han, H. Wang, X. X. He, B. Wang and B. Wu, J. Chromatogr. A, 2016, 1468, 192-199.

19 C. F. Poole and S. K. Poole, J. Chromatogr. A, 2008, 1184, 254280.

20 B. Shaik, J. H. Park, T. K. An, Y. R. Noh, S. B. Yoon, C. E. Park, Y. J. Yoon, Y. H. Kim and S. G. Lee, Tetrahedron, 2013, 69, 8191-8198.

21 S. H. Zeng, L. X. Yin, X. Y. Jiang, Y. Q. Li and K. C. Li, Dyes Pigm., 2012, 95, 229-235.

22 A. J. Huang, N. Nan, M. Chen, X. Pu, H. Tang and Y. L. Sun, Acta Sci. Nat. Univ. Pekin., 1988, 24, 425-429.

23 M. Ge and L. Yao, Spectrochim. Acta, Part A, 2008, 71, 14991502.

24 M. H. Kolář and P. Hobza, Chem. Rev., 2016, 116, 5155-5187.

25 T. Kortvelyesi, M. Gorgenyi and L. Seres, Chromatographia, 1995, 41, 282-286. 